\title{
Les besoins trophiques et thermiques des larves de la coccinelle Harmonia axyridis Pallas
}

\author{
Henrique SCHANDERL, André FERRAN $\left({ }^{*}\right)$ \& Marie-Madeleine LARROQUE (*) \\ Université des Açores, Département de Biologie, PT, 9502 Ponta Delgada \\ (*) I.N.R.A., Station de Zoologie et de Lutte biologique, 37, boulevard du Cap, F 06602 Antibes
}

RÉSUMÉ

\begin{abstract}
Durant sa vie larvaire, la coccinelle Harmonia axyridis Pallas consomme environ $98 \mathrm{mg}$ du puceron $M$ Mzus persicae Sulz. et présente un gain de poids de $40 \mathrm{mg}$. Le rendement alimentaire moyen calculé à l'issue du développement larvaire est de 43 p. 100. Chaque stade larvaire est caractérisé par une relation linéaire entre la consommation alimentaire cumulée et le poids corporel correspondant. Ces besoins trophiques et ces aptitudes physiologiques sont comparables à ceux mis en évidence chez une autre coccinelle de grande taille, Semiadalia undecimnotata Schn.

De même, les exigences thermiques des larves ne sont pas notablement différentes de celles trouvées chez d'autres coccinelles aphidiphages. Sur l'ensemble de la vie larvaire, le seuil thermique théorique est de $10,5^{\circ} \mathrm{C}$ et la constante thermique de 231,3 degrés-jours. L'optimum thermique de croissance se situe entre 20 et $25^{\circ} \mathrm{C}$, températures pour lesquelles la mortalité est minimale.
\end{abstract}

Mots clés additionnels : Coccinellidae, consommation alimentaire, température minimale, constante thermique.

The trophic and thermal requirements of larvae of the ladybeetle Harmonia axyridis Pallas.

During the larval period, the ladybeetle Harmonia axyridis Pallas was found to ingest approximately $98 \mathrm{mg}$ of the aphid Myzus persicae Sulz. for a weight increase of about $40 \mathrm{mg}$. The mean assimilation rate computed at the end of the larval development was $43 \%$. Each larval stage was characterized by a linear relationship between the cumulative food consumption and the corresponding body weight. These trophic requirements and physiological fitnesses were comparable with those found in an other large ladybeetle Semiadalia undecimnotata Schn. and the thermal requirements of the larvae were not very different from those described for some other aphidophagous ladybeetles. The theoretical temperature threshold $(t)$ for all larval cycles was $10.5^{\circ} \mathrm{C}$. Total development required 231.3 heat degree days (K). Mortality was highest at 15 and $30^{\circ} \mathrm{C}$. The optimum temperature was situated between 20 and $25^{\circ} \mathrm{C}$.

Additional key words : Coccinellidae, food consumption, minimal temperature, thermal constant.

\section{INTRODUCTION}

La coccinelle Harmonia axyridis Pallas est originaire d'Asie. Elle se rencontre dans la strate végétale composée par des arbres spontanés ou cultivés (IABLOKOFF-KHNZORIAN, 1982). Son régime alimentaire est essentiellement aphidiphage bien qu'elle ait été observée dans des populations de psylles et de cochenilles. Parmi la dizaine d'espèces de pucerons qu'elle attaque, Myzus persicae Sulz. constitue une proie de très bonne valeur alimentaire (HUKUSIMA \& KAMEI, 1970).

Ce prédateur de grande taille, se caractérise par une importante variabilité de la coloration élytrale
(Komal, 1956), par une fécondité exceptionnelle, comprise entre 2500 et 3800 œufs selon la nourriture ingérée (HUKUSIMA \& KAMEI, 1970) et par une résistance exceptionnelle au froid à l'état adulte (VORONIN, 1966).

Elle a été introduite avec plus ou moins de succès aux îles Hawaï, en Californie et en Sibérie (IABLOKOFF-KHNZORIAN, 1982). Les travaux présentés dans ce texte concernent la quantification de l'efficacité prédatrice des larves et la détermination de leurs caractéristiques thermiques; ils constituent la première étape d'un programme dont l'objectif est l'introduction de cette espèce en France et aux Açores (Portugal). 


\section{MATÉRIEL ET MÉTHODES}

L'étude des besoins trophiques et thermiques des larves de cette coccinelle a été réalisée à l'aide de 2 expériences successives.

\section{A. La détermination des besoins trophiques}

Les larves, élevées en cage individuelle, reçoivent 2 fois par jour, le matin et en fin d'après-midi, un nombre déterminé de pucerons ( $M$. persicae) préalablement pesés. Les jeunes larves ( $1^{\text {er }}$ stade) sont mises en présence de petits pucerons d'âge indéterminé tandis que les stades ultérieurs sont nourris avec des femelles aptères. Les rations alimentaires qui leur sont offertes au début de chaque intervalle de temps sont largement excédentaires, soit, dans l'ordre chronologique du développement, 30 jeunes proies (matin : 10, après-midi : 20$), 40(20,20), 50(20,30)$ et $80(30,50)$ femelles aptères de puceron.

A l'occasion de chaque renouvellement de la nourriture, un bilan trophique et pondéral est dressé selon la méthode proposée par FERRAN \& LARROQUE (1977). A partir de la consommation alimentaire exprimée en $\mathrm{mg}$ et du gain de poids réalisé à l'issue de chaque laps de temps, il est possible, pour chaque stade, de quantifier l'évolution pondérale, de préciser les besoins trophiques, de calculer le rendement alimentaire qui est égal au rapport multiplié par 100 entre le gain de poids et la consommation alimentaire correspondante, et d'établir la relation linéaire entre la consommation alimentaire cumulée et le poids frais.

Les larves sont élevées à $20 \pm 1{ }^{\circ} \mathrm{C}$, sous une humidité relative de 60 à 75 p. 100, avec 16 h d'éclairement journalier.

\section{B. L'étude des besoins thermiques}

Des œufs récemment pondus puis les larves et les nymphes qui en proviennent, maintenus en cage individuelle $(\varnothing: 3 \mathrm{~cm}, \mathrm{~h}: 1 \mathrm{~cm})$, sont élevés à 4 températures différentes : $15 \pm 1{ }^{\circ} \mathrm{C}, 20 \pm 1{ }^{\circ} \mathrm{C}, 25 \pm 1{ }^{\circ} \mathrm{C}$ et $30 \pm 1{ }^{\circ} \mathrm{C}$. Les autres conditions abiotiques sont identiques pour ces 4 lots : photopériode comportant $16 \mathrm{~h}$ d'éclairement, humidité relative, 60 à 75 p. 100, stabilisée grâce à l'emploi de solutions salines saturées appropriées à chaque température, nourriture constituée par un mélange des différents stades du puceron $M$. persicae qui est renouvelé 2 fois par jour.

Pour chaque température et pour chaque stade de la vie pré-imaginale nous avons déterminé la mortalité et la durée (D) correspondante de la croissance.

A partir de ce critère de temps, il est possible de calculer pour chaque stade, un certain nombre d'indices de croissance (Davidson, 1944 ; David \& Clavel, 1966 ; CAMPBELL et al., 1974 ; NEUENSCHWANDER, 1975) :

1) La vitesse de développement (V) qui est l'inverse de la durée (D). Cette vitesse est liée à la température (T) par une fonction logistique ou, dans la zone moyenne des températures, par une relation linéaire qui est moins précise que la précédente $: \mathrm{V}=\mathrm{a}_{2}+\mathrm{b}_{2} \mathrm{~T}$.
Comme les larves ont été seulement élevées dans 4 conditions thermiques différentes, nous avons préféré utiliser la relation linéaire calculée à partir des valeurs individuelles.

2) Le seuil thermique (t) inférieur, obtenu par extrapolation de cette droite à $\mathrm{V}=0$.

$$
\mathrm{t}=\frac{\mathrm{a}_{2}}{\mathrm{~b}_{2}} \text {. }
$$

3) La constante thermique (K), exprimée en degréjour. Elle est calculée à partir de la relation : $\mathrm{K}=\mathrm{Di}(\mathrm{Ti}-\mathrm{t})$, avec $\mathrm{Di}$, durée du développement à la température Ti. Comme, pour chaque stade, cette constante varie sensiblement en fonction de la température (UVAROV, 1931 ; SAVESCU, 1965), la valeur proposée dans le texte correspond à la moyenne de celles calculées pour chaque température.

Les résultats sont exprimés par leur moyenne et l'intervalle de confiance correspondant au seuil 5 p. 100.

\section{RÉSULTATS ET DISCUSSION}

\section{A. Les besoins trophiques au cours de la croissance larvaire (tabl. 1)}

La mortalité au cours des différents stades est nulle, exception faite chez les jeunes larves où elle atteint 2,7 p. 100 de l'effectif initial. Le puceron $M$. persicae présente effectivement une bonne valeur alimentaire pour ce prédateur.

La croissance pondérale au sein de chaque stade est un processus continu qui s'arrête environ 1 jour avant la mue suivante en raison de l'interruption des prises alimentaires. Le gain de poids moyen $(39,97 \mathrm{mg})$ effectué sur l'ensemble de la vie larvaire est sensiblement supérieur à celui observé chez Semiadalia undecimnotata Schn. (31,50 mg) (FERRAN \& LARROQUE, 1977). Les larves de dernier stade assurent 76 p. 100 de ce gain de poids. Cette valeur est comparable à celle mise en évidence chez la coccinelle précédente (79 p. 100) et chez Adalia bipunctata L. (74,4 p. 100) (FERRAN et al., 1984).

La consommation alimentaire exprimée en $\mathrm{mg}$ croît en fonction du stade de $2,17 \mathrm{mg}$ à $84,38 \mathrm{mg}$, ce qui représente pour l'ensemble de la vie larvaire l'ingestion de $104,5 \mathrm{mg}$ de pucerons ou de 204 individus. Cette consommation est voisine de celle déterminée chez $S$. undecimnotata $(98,36 \mathrm{mg})$ mais est supérieure à celle réalisée par une espèce de petite taille A. bipunctata $(54,99 \mathrm{mg})$. Les larves au dernier stade prélèvent 81 p. 100 de la consommation alimentaire totale, valeur identique à celles trouvées chez les 2 espèces précédemment citées, 84 et 81 p. 100 respectivement.

Chez $H$. axyridis également, chaque stade larvaire peut être caractérisé par une relation linéaire entre la consommation alimentaire cumulée, $\mathrm{C}$ (en $\mathrm{mg}$ ) et le poids frais $(\mathrm{P})$ correspondant. Ces relations sont définies pour chaque stade dans l'intervalle de poids compris entre le poids à la mue et le poids maximal atteint. 
TABLEAU 1

Principales caractéristiques biologiques et trophiques des larves de la coccinelle Harmonia axyridis Pallas (température :20 $\pm 1{ }^{\circ} \mathrm{C} ;$ humidité relative : 60 à 75 p. 100 ; éclairement 16 h par jour ; nourrilure : Myzus persicae Sulz.).

Main biological and trophic features of Harmonia axyridis Pallas larvae (temperature: $20 \pm 1{ }^{\circ} \mathrm{C}$; relative humidity: 60 to $75 \%$; light: $16 \mathrm{~h}$ per day ; food: Myzus persicae Sulz.).

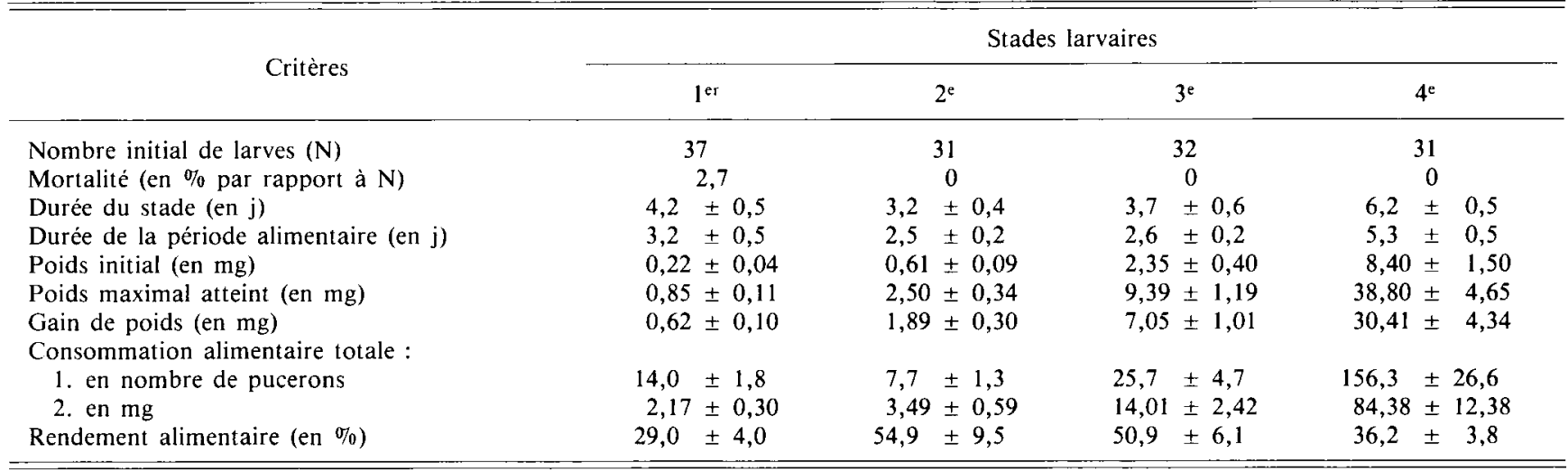

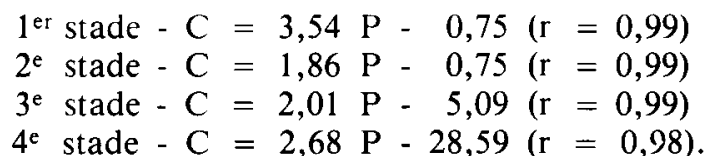

Dans le cas du dernier stade, cette relation peut être également représentée par une fonction puissance à condition que l'origine des poids soit le poids mesuré à l'issue du $1^{\text {er }}$ intervalle et non le poids à la mue :

$$
\mathrm{C}=0,02 \mathrm{P}^{2,3}(\mathrm{r}=0,99) \text {. }
$$

Une telle anomalie a déjà été mise en évidence chez les 2 coccinèlles précédemment citées.

Le rendement alimentaire subit au cours du développement larvaire une évolution identique à celle décrite chez ces 2 espèces : il est maximum au $2^{\mathrm{e}}$ stade puis décroît pour les stades ultérieurs. Sur l'ensemble de la vie larvaire, la valeur moyenne obtenue $(42,7$ p. 100$)$ est voisine de celle calculée chez $S$. undecimnotata $(43,2$ p. 100$)$ et chez $A$. bipunctata $(43,9$ p. 100$)$. Par contre, le rendement alimentaire au cours $\mathrm{du} 1^{\text {er }}$ stade est nettement plus faible 29 p. 100 contre respectivement 44 et 44,3 p. $100-$ tandis qu'il est plus élevé chez les larves âgées 36,2 p. 100 contre 30,4 et 30,5 p. 100 . Comparativement à $S$. undecimnotata, les larves au dernier stade
d'H. axyridis semblent mieux utiliser les proies consommées puisque, à un gain de poids nettement supérieur - 30,41 $\mathrm{mg}$ contre $24,90 \mathrm{mg}$ - correspondent des consommations alimentaires sensiblement identiques $(84,38 \mathrm{mg}$ contre $82,52 \mathrm{mg})$.

\section{B. Les besoins thermiques au cours de la croissance larvaire (tabl. 2)}

La mortalité au cours de la vie larvaire et nymphale décroît quand la température augmente de $15^{\circ} \mathrm{C}$ (50 p. 100 de l'effectif initial $\mathrm{N}$ ) à $25^{\circ} \mathrm{C}$, puis croît de nouveau pour $30^{\circ} \mathrm{C}(14 \mathrm{p} .100)$. Alors qu'à $15^{\circ} \mathrm{C}$ la mortalité, exprimée par rapport au nombre d'individus présents au début de chaque stade, paraît intervenir au niveau des 2 premiers stades ( $1^{\text {er }}: 13,5$ p. 100 ; $\left.2^{\mathrm{e}}: 12,5 \mathrm{p} .100\right)$, à $30^{\circ} \mathrm{C}$, elle affecte plutôt les stades âgés $\left(3^{\mathrm{e}}: 2,8\right.$ p. $100 ; 4^{\mathrm{e}}: 8,8$ p. 100$)$.

Comme le montre le tableau 3 , le poids des adultes issus des larves élevées à $15^{\circ} \mathrm{C}$ est significativement plus faible. Il est, par contre, sensiblement constant dans les autres conditions thermiques.

La durée (D) de la période embryonnaire, celle des différents stades larvaires et de la nymphose sont liées à la température $(\mathrm{T})$ par une relation hyperbolique $\mathrm{du}$

TABLEAU 2

Durée (en jours) du développement de la coccinelle Harmonia axyridis Pallas en fonction de la température (humidité relative : 60 à 75 p. 100 ; $16 \mathrm{~h}$ d'éclairement par jour; nourriture: Myzus persicae Sulz.).

Time (in days) required for development of Harmonia axyridis Pallas under constant temperature (relative humidity : 60 to $75 \%$; light : I6 h per day ; food: Myzus persicae Sulz.).

\begin{tabular}{|c|c|c|c|c|}
\hline \multirow{2}{*}{$\begin{array}{c}\text { Stades } \\
\text { pré-imaginaux }\end{array}$} & \multicolumn{4}{|c|}{ Températures $\left(\mathrm{en}{ }^{\circ} \mathrm{C}\right)$} \\
\hline & 15 & 20 & 25 & 30 \\
\hline Nombre initial d'individus & 37 & 30 & 35 & 35 \\
\hline Oeuf & $9,2 \pm 0.1$ & $4,4 \pm 0,1$ & $3,4 \pm 0,1$ & $2,1 \pm 0,1$ \\
\hline $1^{\text {er }}$ stade & $6,7 \pm 0,6$ & $4,2 \pm 0,2$ & $1,9 \pm 0,1$ & $1,8 \pm 0,2$ \\
\hline $2^{e}$ stade & $6,1 \pm 0,3$ & $2,5 \pm 0,2$ & $1,9 \pm 0,1$ & $1,2 \pm 0,1$ \\
\hline $4^{e}$ stade & $11,8 \pm 0,7$ & $6,2 \pm 0,4$ & $3,7 \pm 0,1$ & $3,4 \pm 0,2$ \\
\hline $4^{e}$ stade fixé & $3,5 \pm 0,4$ & $1,4 \pm 0,1$ & $0,9 \pm 0,1$ & $0,5 \pm 0,0$ \\
\hline Nymphe & $18,7 \pm 0,6$ & $7,9 \pm 0,1$ & $4,9 \pm 0,1$ & $3,9 \pm 0,1$ \\
\hline Durée totale du développement & $51,0 \pm 1,3$ & $25,2 \pm 0,7$ & $15,2 \pm 0,3$ & $12,1 \pm 0,3$ \\
\hline
\end{tabular}


TABLEAU 3

Poids moyen des adultes de la coccinelle Harmonia axyridis Pallas provenant de larves élevées à différentes températures.

Mean weight of Harmonia axyridis Pallas adults from larvae bred at different temperatures.

\begin{tabular}{ccc}
\hline \multirow{2}{*}{$\begin{array}{c}\text { Températures } \\
\left({ }^{\circ} \mathrm{C}\right)\end{array}$} & \multicolumn{2}{c}{ Poids des adultes (en mg) } \\
\cline { 2 - 3 } & Femelles & Mâles \\
\hline 15 & $23,26 \pm 2,48$ & $22,35 \pm 2,68$ \\
20 & $31,97 \pm 1,16$ & $26,69 \pm 1,18$ \\
25 & $30,52 \pm 2,16$ & $26,90 \pm 1,62$ \\
30 & $32,24 \pm 2,55$ & $28,69 \pm 2,11$ \\
\hline
\end{tabular}

type :

$$
\mathrm{D}=\mathrm{a}_{1}+\frac{\mathrm{b}_{1}}{\mathrm{~T}-\mathrm{c}_{1}}(\text { tabl. 4) }
$$

avec $a_{1}$ : la durée minimale théorique du développement qui est atteinte lorsque la température augmente

$b_{1}$ : l'accélération de la vitesse de développement

$c_{1}$ : le seuil thermique inférieur.

Dans la gamme thermique considérée, les relations linéaires entre les vitesses (V) de développement au cours des différents stades pré-imaginaux et la température sont toutes significatives au seuil 5 p. 100 (tabl. 4). De telles relations ont été décrites chez plusieurs autres coccinelles aphidiphages par OBRYCKI \& TAUBER (1981). Toutefois, en élevant des larves de Coccinella septempunctata $\mathrm{L}$. dans une gamme de températures plus large, HoDEK (1958) a trouvé des relations logistiques normales.

Les seuils thermiques mis en évidence à partir des durées de développement (fonction hyperbolique) sont, pour certains stades $\left(1^{\mathrm{er}}, 3^{\mathrm{e}}\right.$ et $\left.4^{\mathrm{e}}\right)$ sensiblement différents de ceux calculés à partir des vitesses (fonction linéaire). Il est très probable que l'estimation des seuils thermiques à partir de valeurs asymptotiques est moins précise que celle obtenue par extrapolation de droites.
Les seuils thermiques théoriques ( $\mathrm{t}$ en ${ }^{\circ} \mathrm{C}$ ) trouvés à partir des relations linéaires chez $H$. axyridis (tabl. 4 et fig. 1) varient de 8,6 à $12,5{ }^{\circ} \mathrm{C}$ selon le stade considéré et sont de ce fait comparables à ceux mis en évidence chez Coccinella transversoguttata Brown.,
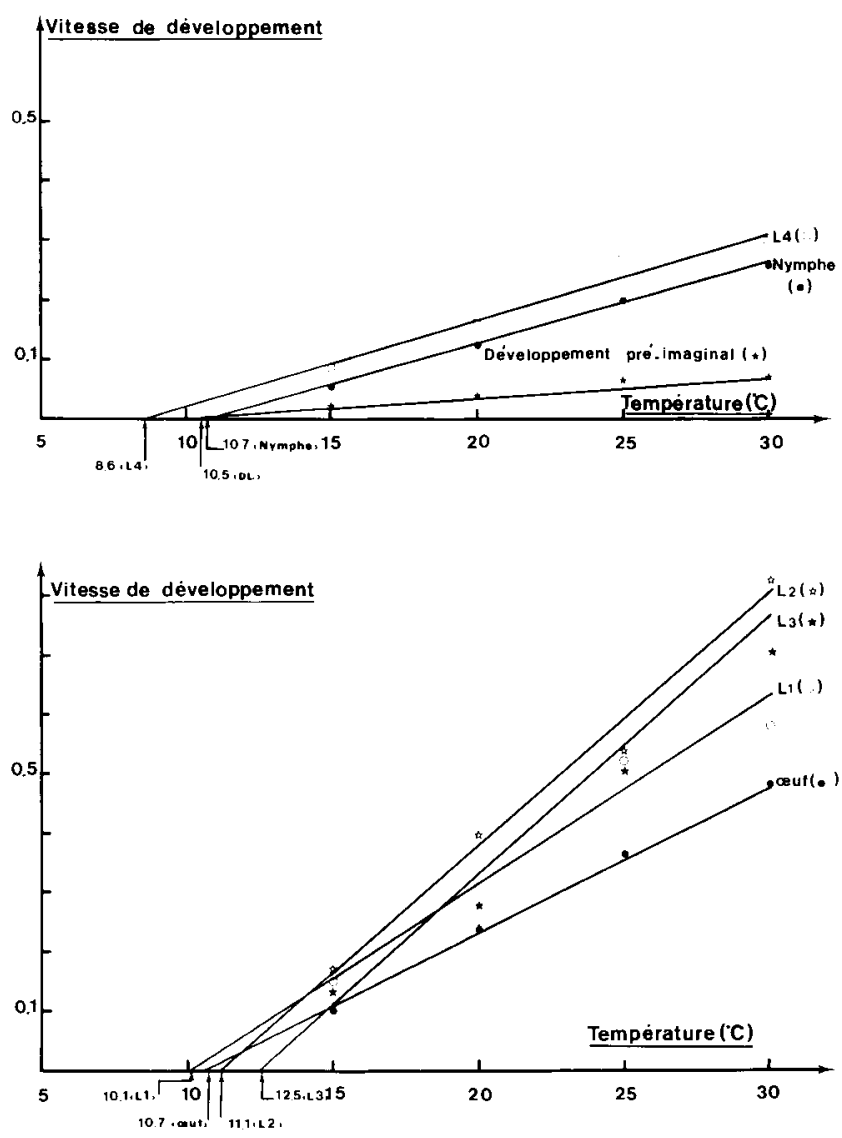

Figure 1

Relations linéaires entre la vitesse de développement et la température chez les reufs et les différents stades larvaires de la coccinelle Harmonia axyridis Pallas (les seuils thermiques sont indiqués par des petits nombres, les symboles représentent les vitesses moyennes par stade et par température).

Linear regressions between growth rate and temperature for the eggs and differents instars of the lady-beetle Harmonia axyridis Pallas $(t$ values in small numbers; the symbols represent average rate per instar and per temperature).

TABLEAU 4

Principales caractéristiques thermiques des larves de la coccinelle Harmonia axyridis Pallas calculées à partir de leur élevage à 4 températures : $15{ }^{\circ} \mathrm{C}, 20{ }^{\circ} \mathrm{C}, 25{ }^{\circ} \mathrm{C}$ et $30{ }^{\circ} \mathrm{C}$; (éclairement $16 \mathrm{~h}$ par jour ; nourriture: Myzus persicae Sulz. ; * coefficient de corrélation). Main thermal features of Harmonia axyridis Pallas computed from their breeding at 4 temperatures:

$15{ }^{\circ} \mathrm{C}, 20{ }^{\circ} \mathrm{C}, 25{ }^{\circ} \mathrm{C}$ and $30^{\circ} \mathrm{C}$; (light: $16 \mathrm{~h}$ per day; food: Myzus persicae Sulz. ; * coefficient of determination).

\begin{tabular}{|c|c|c|c|c|c|c|c|}
\hline \multirow[t]{2}{*}{ Stade } & \multicolumn{3}{|c|}{$\begin{array}{l}\text { Durée (D) du développement } \\
\qquad b_{1}\end{array}$} & \multicolumn{2}{|c|}{$\begin{array}{l}\text { Vitesse (V) du développement } \\
\qquad V=a_{2}+b_{2} T\end{array}$} & \multirow{2}{*}{$\begin{array}{l}\text { Seuil thermique } \\
\text { théorique } \\
\text { (t en }{ }^{\circ} \mathrm{C} \text { ) }\end{array}$} & \multirow{2}{*}{$\begin{array}{l}\text { Constante } \\
\text { thermique } \\
\left(\mathrm{K} \text { en }{ }^{\circ} \mathrm{J}\right)\end{array}$} \\
\hline & $a_{1}$ & $b_{1}$ & $\mathrm{c}_{1}$ & $a_{2}$ & $b_{2}$ & & \\
\hline Oeufs & 0,44 & 37,1 & $10,7(0,98)^{*}$ & $-0,25$ & $0,024(0,94)$ & 10,7 & $42,4 \pm 4,1$ \\
\hline $1^{\text {er }}$ stade & $-1,60$ & 75,9 & $5,6(0,91)$ & $-0,33$ & $0,032(0,83)$ & 10,1 & $34,7 \pm 5,5$ \\
\hline $2^{\mathrm{e}}$ stade & 0,29 & 19,2 & $11,7(0,96)$ & $-0,48$ & $0,043(0,88)$ & 11,1 & $23,8 \pm 1,8$ \\
\hline $3^{e}$ stade & $-1,27$ & 54,9 & $8,5(0,97)$ & $-0,55$ & $0,044(0,89)$ & 12,5 & $23,3 \pm 3,7$ \\
\hline $4^{e}$ stade & 0,43 & 54,2 & $10,2(0,95)$ & $-0,13$ & $0,015(0,92)$ & 8,6 & $69,9 \pm 6,3$ \\
\hline Nymphe & 0,47 & 61,2 & $11,6(0,99)$ & $-0,15$ & $0,014(0,98)$ & 10,7 & $74,8 \pm 4,2$ \\
\hline Total & $-1,63$ & 262,2 & $10,1(0,99)$ & $-0,046$ & $0,004(0,94)$ & 10,5 & $231,3 \pm 8,2$ \\
\hline
\end{tabular}


C. septempunctata (OBRYCKI \& TAUBER, 1981) et Coleomegilla maculata De Geer (OBRYCKI \& TAUBER, 1978). A titre d'exemple, le seuil thermique pour l'ensemble du développement pré-imaginal est, dans l'ordre des espèces citées, de $12,2-12,1$ et $11,3^{\circ} \mathrm{C}$ alors qu'il est de $10,5^{\circ} \mathrm{C}$ chez $H$. axyridis. Les principales différences se situent au niveau des œufs chez C. septempunctata $\left(\mathrm{t}=6,8^{\circ} \mathrm{C}\right)$ et de l'ensemble $\mathrm{du}$ développement larvaire chez $A$. bipunctata $\left(\mathrm{t}=9,0^{\circ} \mathrm{C}\right)$ et chez Hemerobius pacificus Banks. $\left(\mathrm{t}=0,6^{\circ} \mathrm{C}\right)$ (NEUENSCHWANDER, 1975).

De même, la constante thermique $\left(\mathrm{K}=231,3^{\circ}-\mathrm{j}\right)$ est voisine de celle mise en évidence par ces auteurs chez $A$. bipunctata $\left(262,3^{\circ}-\mathrm{j}\right), C$. transversoguttata $\left(218,2^{\circ}-\mathrm{j}\right)$, C. septempunctata $\left(197^{\circ}-\mathrm{j}\right)$ et $C$. maculata $\left(235,8^{\circ}-\mathrm{j}\right)$ mais elle est nettement inférieure à celle déterminée chez $H$. pacificus $\left(547,8^{\circ}-\mathrm{j}\right)$.

\section{CONCLUSION}

Les larves de la coccinelle $H$. axyridis ne se distinguent pas particulièrement des autres espèces étudiées aussi bien au niveau des besoins trophiques que des besoins thermiques.

Bien que leur poids à la fin du développement soit supérieur à celui obtenu chez $S$. undecimnotata, la consommation alimentaire demeure comparable en raison notamment d'un meilleur rendement alimentaire au cours du dernier stade larvaire. Les différents stades larvaires sont également caractérisés par une relation linéaire entre la consommation alimentaire cumulée et le poids corporel frais correspondant.
Exposées à des températures basses, les larves donnent naissance à des adultes de plus petite taille. En raison de l'existence des relations précédentes, on peut penser que leur activité trophique est globalement réduite dans ces conditions thermiques. Chez $S$. undecimnotata au contraire, la consommation alimentaire totale et, par conséquent, le poids des larves et des adultes qui en proviennent, diminuent quand la température augmente (FERRAN \& LARROQUE, 1979).

Dans la mesure où les résultats obtenus en laboratoire peuvent être appliqués sur le terrain, la connaissance du seuil thermique inférieur permet de prévoir la précocité d'une espèce tandis que la constante thermique offre la possibilité d'estimer le nombre de générations par an en fonction notamment des températures maximales et minimales relevées (BASKERVILLE \& EMIN, 1969). Ces 2 aspects sont intéressants à considérer lorsqu'on se propose d'introduire une nouvelle espèce.

Compte tenu de la répartition de la mortalité en fonction de la température, la zone thermique optimale pour cette espèce semble se situer entre 20 et $25{ }^{\circ} \mathrm{C}$.

Reçu le 23 mai 1984. Accepté le 17 décembre 1984.

\section{REMERCIEMENTS}

Nous remercions M. IPERTI (I.N.R.A.-Antibes) et M. le Professeur V. Garcia (Université des Açores, Portugal), responsables de ce programme d'acclimatation, pour leur aide et leurs conseils ainsi que M. LAPCHIN (I.N.R.A., Antibes) pour sa participation aux analyses statistiques.

\section{RÉFÉRENCES BIBLIOGRAPHIQUES}

Baskerville G. L., Emin P., 1969. Rapid estimation of heat accumulation from maximum and minimum temperatures. Ecology, 50, 514-517.

Campbell A., Frazer B. D., Gilbert N., Gutierrez A. P., Mackauer M., 1974. Temperature requirements of some aphids and their parasites. J. Appl. Ecol., 11, 431-438.

David J., Clavel M. F., 1966. Essai de définition d'une température optimale pour le développement de la drosophile. C. R. Acad. Sci. Paris, Ser. D, 262, 2159-2162.

Davidson J., 1944. On the relationship between temperature and rate of development of insects at constant temperatures. J. Anim. Ecol., 13, 26-38.

Ferran A., Larroque M. M., 1977. Etude des relations hôteprédateur et de la consommation et l'utilisation d'un puceron Myzus persicae Sulz. par les différents stades larvaires de la coccinelle Semiadalia undecimnotata Schn. Ann. Zool. Ecol. Anim., 9, 665-691.

Ferran A., Larroque M. M., 1979. Influence des facteurs abiotiques sur la physiologie alimentaire des larves de la coccinelle aphidiphage Semiadalia undecimnotata (Col. Coccinellidae). I. Action de la température. Entomophaga, 24, 403-410.

Ferran A., Cruz De Boelpaepe M. O., Buscarlet L. A., Larroque M. M., Schanderl H., 1984. Les relations trophiques entre les larves de la coccinelle Semiadalia undecimnotata Schn. et le puceron Myzus persicae Sulz. Généralisation à d'autres couples «proieprédateur » et influence des conditions d'élevage de l'auxiliaire. Acta Oecol., Oecol. Appl., 5, 85-97.

Hodek I., 1958. Influence of temperature, relative humidity and photoperiodicity on the speed of development of Coccinella septempunctata L. Acta Soc. Entomol. Tchec., 55, 121-141.

Hukusima S., Kamei M., 1970. Effects of various species of aphids as food on development, fecundity and longevity of Harmonia axyridis Pallas (Col., Coccinellidae). Res. Bull. Fac. Agric., Gifu Univ., 29, 53-66.

Iablokoff-Khnzorian S. M., 1982. Les coccinelles, coléoptères Coccinellidae. Soc. Nouv. Ed. Boubé Paris, 568 p.

Komai T., 1956. Genetics of ladybeetles. Adv. Genet., 8, 155-185.

Neuenschwander P., 1975. Influence of temperature and humidity on the immature stages of Hemerobius pacificus. Environ. Entomol., 4, 215-220.

Obrycki J. J., Tauber M. J., 1978. Thermal requirements of development of Coleomegilla maculata (Col., Coccinellidae) and its parasite Perilitus coccinellae (Hym., Braconidae). Can. Entomol., 110, 407-412.

Obrycki J. J., Tauber M. J., 1981. Phenology of three coccinellid species thermal requirements for development. Ann. Entomol. Soc. Am., 74, 31-36.

Savescu A., 1965. Constantele dezvoltarii insectelor polivoltine si importante lor pentru teoria si practica protectici plantelor. Anal. Sect. Prot. Plant., 3, 289-304.

Uvarov B. P., 1931. Insect and climate. Trans. Entomol. Soc. London, 79, 1-247.

Voronin E. K., 1966. Biologie du prédateur des aphidiens, Harmonia axyridis Pallas. Ins. Nuis. de l'Extrême Orient Soviét., 177-185. 